\title{
A Volumetric Magnetic Resonance Imaging Study of the Amygdala in Frontotemporal Lobar Degeneration and Alzheimer's Disease
}

\author{
Jennifer L. Whitwell ${ }^{a, b}$ Elizabeth L. Sampson ${ }^{a, c}$ Hilary C. Watt ${ }^{a, d}$ \\ Richard J. Harvey, ${ }^{a, e}$ Martin N. Rossor ${ }^{a, f}$ Nick C. Fox $^{a}$ \\ ${ }^{a}$ Dementia Research Centre, Institute of Neurology, University College London, London, UK; ${ }^{b}$ Department of \\ Diagnostic Radiology, Mayo Clinic, Rochester, Minn., USA; ${ }^{c}$ Department of Mental Health Sciences, Royal Free \\ and University College Medical School, London, UK; ${ }^{d}$ Medical Statistics Unit, London School of Hygiene and \\ Tropical Medicine, London, UK; ${ }^{e}$ Department of Clinical and Biomedical Science, University of Melbourne, \\ Melbourne, Australia; ${ }^{\mathrm{f}}$ Division of Neuroscience and Psychological Medicine, Imperial College London, \\ London, UK
}

\section{Key Words}

Magnetic resonance imaging $\cdot$ Amygdala .

Frontotemporal lobar degeneration · Alzheimer's disease

\begin{abstract}
The amygdala is severely atrophied at post-mortem in frontotemporal lobar degeneration (FTLD), and may contribute to the prominent behavioural changes that are early features of FTLD. The aim of this study was to assess amygdala atrophy using MRI in the main syndromic variants of FTLD and Alzheimer's disease (AD). Brain and amygdala volumes, adjusted for intracranial volume, were measured on 46 clinically diagnosed FTLD patients [22 frontal variant FTD (FTD), 14 semantic dementia (SD), 10 progressive non-fluent aphasia (PNFA)], 20 AD patients, and 17 controls. While severe amygdala atrophy was present in both FTLD ( $41 \%$ smaller than controls on the left; $33 \%$ on the right) and in $A D(22 \%$ on the left; $19 \%$ on the right), the FTLD group had significantly greater amygdala atrophy $(z=3.21, p=0.001$ left, $z=2.50$, $p=0.01$ right $)$ and left/right asymmetry $(z=2.03, p=0.04)$ than AD. Amygdala atrophy was greater in SD than FTD, PNFA and AD ( $p<0.02$ for all). Highly asymmetrical atrophy was present in $S D$, greater on the left $(z=3.23$,
\end{abstract}

$p=0.001$ ), and to a lesser extent in PNFA. Despite an overlap between clinical and radiological features of FTLD and AD, marked amygdala atrophy points towards a diagnosis of FTLD, with left greater than right atrophy suggestive of one of the language variants.

Copyright $(2005$ S. Karger AG, Basel

\section{Introduction}

The frontotemporal lobar degenerations (FTLD) are a group of progressive degenerative disorders associated with focal degeneration of the frontal and temporal lobes. These diseases are a common cause of early-onset dementia [1] and are characterised clinically by early behavioural change, with a relative sparing of episodic memory. FTLD has been subdivided clinically into three main syndromic variants: frontotemporal dementia (FTD), semantic dementia (SD) and progressive non-fluent aphasia (PNFA) [2]. FTD patients present with early and prominent behavioural problems, including alterations in personality and social conduct. While PNFA and SD are both characterised by prominent and early language deficits, behavioural features often develop with progression of the syndrome [2].

\section{KARGER}

Fax +41613061234 E-Mail karger@karger.ch www.karger.com
(C) 2005 S. Karger AG, Basel $1420-8008 / 05 / 0204-0238 \$ 22.00 / 0$

Accessible online at:

www.karger.com/dem
Prof. Nick Fox

Dementia Research Centre, National Hospital for Neurology and Neurosurgery

8-11 Queen Square, London WC1N 3BG (UK)

Tel. +44 207829 8773, Fax +44 2076762066

E-Mailnfox@dementia.ion.ucl.ac.uk 
Animal and human studies have shown that damage to the amygdala is associated with prominent behavioural changes, including altered dietary habits, prominent oral tendencies and hypersexuality [3, 4]. Emotional agnosia is also a common feature of FTLD [5] and has been associated with amygdala damage [6]. MRI studies have demonstrated significant atrophy of the amygdala in patients with SD [7-10] and FTD [11], and pathological studies have shown that the amygdala is one of the most severely affected structures in Pick's disease [3, 12], an important pathological substrate of FTLD.

Differentiating FTLD from Alzheimer's disease (AD) can be difficult and although AD is characterised by early memory decline there may be considerable overlap in clinical features between AD and FTLD, especially in the early stages. Behavioural features are also common in AD [13]. Accurate diagnosis is important since each has a different clinical course and specific treatments for $\mathrm{AD}$ are now available. While amygdala atrophy has been demonstrated on MRI in AD [14], it is unclear if it is useful in the differential diagnosis of $\mathrm{AD}$ [15], and there are no volumetric MRI studies comparing amygdala atrophy in all three variants of FTLD to AD. The aim of this study was to investigate amygdala atrophy in all three syndromic variants of FTLD, and to determine whether amygdala atrophy may be useful in the differential diagnosis of FTLD and AD.

\section{Materials and Methods}

\section{Subjects}

All subjects were recruited retrospectively from a specialist cognitive disorders clinic with ethics committee approval and written consent from all study participants. A detailed history, neurological and general examination, screening blood tests, neuropsychometry, including the Mini-Mental State Examination (MMSE) [16], and MR imaging had been performed for all subjects. Disease duration was defined as the time from first symptom onset to MRI scan. Forty-six patients fulfilling consensus criteria for FTLD [2] were included. Twenty-two of these patients were diagnosed with FTD (often referred to as frontal variant FTD), 14 with SD, and 10 with PNFA. Post-mortem confirmation was available in 4 cases (2 Pick's disease and 2 FTLD with ubiquitin-only-immunoreactive neuronal changes). The cohort was age- and gender-matched to 20 patients that fulfilled the NINCDS-ADRDA criteria for 'probable' AD [17] (8 familial, 12 sporadic), and 17 healthy controls. The control subjects were typical of normal ageing and had no complaints of cognitive impairment or behavioural problems. Exclusion criteria included a history of stroke or dementia. MRI was used to exclude space-occupying lesions, vascular disease and other pathologic conditions; however, the diagnosis of FTLD or $\mathrm{AD}$ was made purely on clinical grounds without further reference to MRI.

\section{Image Analysis}

$\mathrm{T}_{1}$-weighted volumetric MR images were acquired on a 1.5tesla Signa unit (GE, Milwaukee, Mich., USA) using a spoiled gradient echo technique (matrix $256 \times 128 \times 128$; field of view, 24 $\times 24 \times 19.2 \mathrm{~cm}$; TR, $35 \mathrm{~ms}$; TE, $5 \mathrm{~ms}$; NEX, 1; flip angle, $35^{\circ}$ ), yielding 124 contiguous $1.5-\mathrm{mm}$ coronal slices with in-plane voxel dimensions of $0.9375 \times 0.9375 \mathrm{~mm}$. All measurements were performed using MIDAS image analysis software [18], which allows simultaneous image viewing and outlining of regions in coronal, sagittal and axial orientations. All measurements were performed blind to subject details and the results of any other measurements.

Brain volumes were obtained using a semi-automated iterative three-dimensional morphological technique that has been described previously [18]. The left and right amygdalae were outlined manually starting from the anterior limit, defined as the anterior extreme of the temporal stem. Posteriorly the amygdala was defined using the border with the lateral ventricle and the alveus. Superiorly the boundary was set anteriorly by a line connecting the inferior point of the lateral fissure to the lateral most point of the paramedian cisternae and posteriorly by the superior and lateral borders of the optic tract. Inferolaterally the amygdala was bounded by white matter and medially by the ambient cistern [8]. An intensity threshold of $70 \%$ mean brain signal intensity was used for consistent segmentation of cerebrospinal fluid from brain. Scans were flipped about the mid-sagittal plane ensuring that the rater was blinded to left/right orientation. The same rater performed all amygdala measurements; their intra-rater ICC (intra-class correlation coefficient) on repeating 12 measurements was $0.98(95 \% \mathrm{CI}$ $0.97-1.00)$; this relates to an average $4 \%$ difference between the repeated measurements of volume.

\section{Statistics}

All volumes were normalised to total intracranial volume (TIV) to reduce the effect of inter-individual variation in head size [19]. Both amygdala and brain volumes were regressed against TIV in the control group to establish the slope of the relationship with TIV. All volumes in the FTLD, AD and control groups were then standardised to TIV volume using the following equation:

$$
\mathrm{sbv}=\mathrm{bv}-\beta(\mathrm{TIV}-\text { meanTIV })
$$

$\mathrm{sbv}=$ standardised volume, $\mathrm{bv}=$ crude volume, $\beta=$ regression coefficient of volume on TIV in controls, mean TIV = mean TIV in the control group.

Amygdala asymmetry score was calculated as follows:

$$
\text { Asymmetry score }=\frac{(\text { left }- \text { right }) \times 2}{\text { left }+ \text { right }}
$$

Age, MMSE and volume measurements were compared between groups using the Mann-Whitney U test; average percentage volume changes were reported based on analysis of log volumes. Proportions were compared using the Fisher's exact test. The Wilcoxon signed rank test was used to compare left and right amygdala volumes. Sensitivity and specificity were calculated from logistic regression and used to determine whether amygdala volume and asymmetry could distinguish between patients with FTLD (and its subgroups) from controls, and secondly, from AD. 
Table 1. Patient characteristics

\begin{tabular}{|c|c|c|c|c|c|c|}
\hline & \multicolumn{3}{|c|}{ Disease groups } & \multicolumn{3}{|l|}{ FTLD groups } \\
\hline & $C(n=17)$ & $\mathrm{AD}(\mathrm{n}=20)$ & $\operatorname{FTLD}(n=46)$ & FTD $(n=22)$ & $\operatorname{PNFA}(n=10)$ & $\mathrm{SD}(\mathrm{n}=14)$ \\
\hline Subjects, male/total ( $\%$ of total) & 9/17 (52.9) & $12 / 20(60.0)$ & $32 / 46(69.6)$ & $18 / 22(81.8)$ & $6 / 10(60.0)$ & $8 / 14(57.1)$ \\
\hline Age, mean years ${ }^{\mathrm{c}}$ & $63.6(10.5)$ & $63.8(9.1)$ & $64.0(6.6)$ & $64.0(6.0)$ & $62.5(8.7)$ & $65.2(6.3)$ \\
\hline $\operatorname{MMSE}(\max 30)$, mean $^{c}$ & $28.9(1.3)$ & $20.3(5.1)^{\mathrm{a}}$ & $19.8(9.2)^{\mathrm{a}}$ & $24.0(6.5)^{\mathrm{a}, \mathrm{b}}$ & $20.1(8.3)^{\mathrm{a}}$ & $12.2(10.2)^{\mathrm{a}, \mathrm{b}}$ \\
\hline Disease duration, mean years ${ }^{\mathrm{c}}$ & N/A & $4.5(2.4)$ & $5.3(2.7)$ & $5.7(3.1)$ & $4.7(2.6)$ & $5.1(2.2)$ \\
\hline
\end{tabular}

$\mathrm{C}=$ Controls; $\mathrm{N} / \mathrm{A}=$ not applicable.

Significant difference from controls using Mann-Whitney U, or Fisher's exact test: ${ }^{a} p<0.01$.

Significant difference from AD using the Mann-Whitney U, or Fisher's exact test: ${ }^{\mathrm{b}} \mathrm{p}<0.05$.

${ }^{\mathrm{c}}$ Means, standard deviation in parentheses.

Table 2. Volumetric results

\begin{tabular}{|c|c|c|c|c|c|c|}
\hline & \multicolumn{3}{|c|}{ Disease groups } & \multicolumn{3}{|l|}{ FTLD groups } \\
\hline & $C(n=17)$ & $\mathrm{AD}(\mathrm{n}=20)$ & FTLD $(n=46)$ & $\operatorname{FTD}(\mathrm{n}=22)$ & $\operatorname{PNFA}(n=10)$ & $\operatorname{SD}(n=14)$ \\
\hline Brain & $1,179(74)$ & $1,102(46)^{b}$ & $1,092(70)^{\mathrm{b}}$ & $1,124(68)^{\mathrm{a}}$ & $1,050(40)^{\mathrm{b}, \mathrm{c}}$ & $1,071(70)^{\mathrm{b}}$ \\
\hline Left amygdala & $1.64(0.21)$ & $1.29(0.24)^{\mathrm{b}}$ & $1.02(0.33)^{\mathrm{b}, \mathrm{d}}$ & $1.18(0.31)^{\mathrm{b}}$ & $1.11(0.21)^{\mathrm{b}, \mathrm{c}}$ & $0.70(0.21)^{\mathrm{b}, \mathrm{d}}$ \\
\hline Right amygdala & $1.68(0.31)$ & $1.35(0.26)^{b}$ & $1.15(0.32)^{b, c}$ & $1.21(0.35)^{b}$ & $1.28(0.30)^{\mathrm{b}}$ & $0.97(0.22)^{b, d}$ \\
\hline Absolute asymmetry & $0.10(0.07)$ & $0.11(0.07)$ & $0.20(0.18)^{\mathrm{a}, \mathrm{c}}$ & $0.12(0.10)$ & $0.17(0.17)$ & $0.35(0.21)^{\mathrm{b}, \mathrm{d}}$ \\
\hline
\end{tabular}

$\mathrm{C}=$ Controls. All volumes are normalised by TIV and expressed in millilitres (mean, standard deviation in parentheses).

Significant difference from controls using the Mann-Whitney U test: ${ }^{\mathrm{a}} \mathrm{p}<0.05,{ }^{\mathrm{b}} \mathrm{p}<0.01$.

Significant difference from AD using the Mann-Whitney U test: ${ }^{c} p<0.05,{ }^{d} p<0.01$.

\section{Results}

There were no significant differences in gender or age distribution between the disease groups and controls, or between the different FTLD diagnostic subgroups (table 1). There was no significant difference in disease duration between FTLD and AD or between the different FTLD diagnostic subgroups. AD and FTLD groups had similar mean MMSE scores.

TIV-adjusted brain volumes were similarly significantly reduced by $6 \%(95 \%$ CI $3-10 \%)$ in AD compared with controls $(z=3.32, p=0.0009)$ and $7 \%(4-11 \%)$ in FTLD compared with controls $(z=3.72, p=0.0002)$. No significant difference was found between FTLD and AD groups $(\mathrm{z}=0.66, \mathrm{p}=0.51$, table 2$)$.

Figure 1 shows examples of representative amygdalae from each group. Compared with controls TIV-adjusted amygdala volumes were significantly reduced by $41 \%$ $(29-51 \%)$ on the left and $33 \%(22-43 \%)$ on the right in the FTLD group, and by $22 \%(13-31 \%)$ on the left and $19 \%(8-29 \%)$ on the right in the AD group. Both left and right amygdala volumes were significantly smaller in the FTLD group than the AD group (left $\mathrm{z}=3.21, \mathrm{p}=0.001$, right $\mathrm{z}=2.50, \mathrm{p}=0.01$ ), even when brain volume was accounted for (left $\mathrm{z}=3.07, \mathrm{p}=0.001$, right $\mathrm{z}=2.36, \mathrm{p}=$ $0.02)$. Significant amygdala asymmetry was present only in the FTLD group, with left amygdala volumes on average $13 \%(6-18 \%)$ smaller than right $(\mathrm{z}=3.47, \mathrm{p}<0.001$, fig. 2a). The absolute amygdala asymmetry score in FTLD was $0.20 \pm 0.18$ (mean \pm standard deviation), which was significantly greater than the score for the $\mathrm{AD}$ group, 0.11 $\pm 0.07(\mathrm{z}=1.97, \mathrm{p}=0.04)$, and the control group, 0.10 $\pm 0.07(z=2.03, p=0.04)$.

In each of the FTLD subgroups the mean left and right amygdala volumes were significantly smaller than control volumes ( $p<0.007$ for all, table 2$)$. The SD group had the smallest amygdala volumes, significantly smaller than the AD, FTD and PNFA groups ( $p<0.02$ for all, left and right). The left amygdala in the SD group was reduced to less than half the mean control volume; this 59\% (51$65 \%$ ) reduction on the left was significantly greater than the right amygdala volume reduction of $45 \%(33-54 \%)$ of 

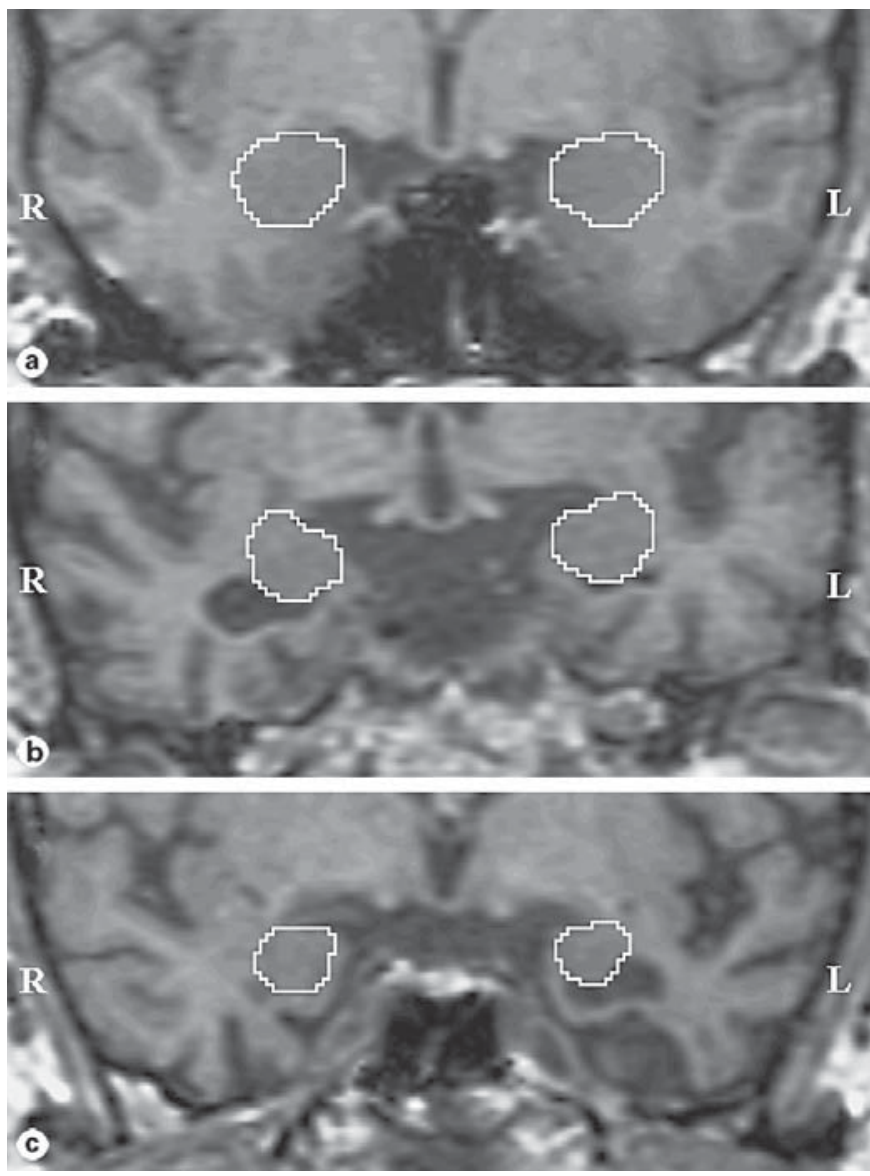

Fig. 1. Coronal sections through the temporal lobes in a control subject (a), a patient with AD (b), and a patient with FTLD (SD variant) $(\mathbf{c})$. Both left and right amygdalae have been outlined. $\mathrm{L}=$ Left; $\mathrm{R}$ = right.
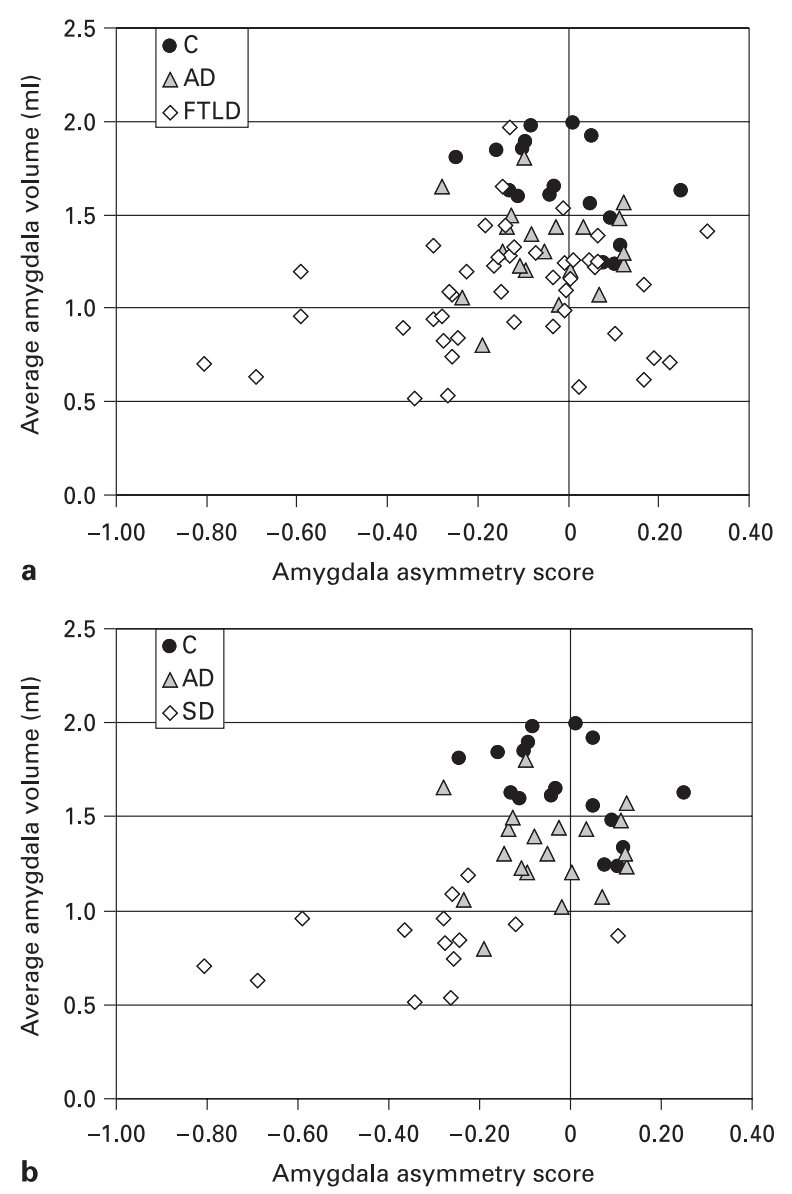

Fig. 2. Average amygdala volume against amygdala asymmetry score in controls (C), AD and either FTLD (a) or SD (b) groups.
Table 3. Screening performance by disease type according to cut-off of average amygdala volume (average of left and right volumes)

\begin{tabular}{lrrrr}
\hline Cut-off level used on average amygdala volume, $\mathrm{mm}^{3}$ & 1,000 & 1,055 & 1,200 & 1,260 \\
\hline Sensitivity (\%) in FTLD (n = 46) & 35 & 41 & 57 & 63 \\
Sensitivity (\%) in FTD ( $=22)$ & 18 & 27 & 45 & 55 \\
Sensitivity (\%) in PNFA (n=10) & 10 & 10 & 30 & 40 \\
Sensitivity (\%) in SD (n = 14) & 79 & 86 & 93 & 93 \\
Specificity (\%) in controls ( $=17)$ & 100 & 100 & 94 & 88 \\
Specificity (\%) in AD (n = 20) & 85 & 85 & 60 & 60 \\
\hline
\end{tabular}

Sensitivity = Percentage of patients with average amygdala measurement at or below the specified cut-off level; specificity = percentage of patients with average amygdala measurement above the specified cut-off level; $\mathrm{C}=$ controls. 
mean control volume $(\mathrm{z}=3.23, \mathrm{p}=0.001)$. The PNFA group also had asymmetrical amygdala volumes, with greater left-sided atrophy, although this was not significant $(z=1.68, p=0.09)$. Symmetrical atrophy was observed in the frontal variant FTD group (fig. $2 b$ ).

Average (mean of left and right) amygdala volumes discriminated between FTLD and controls with a specificity of $88 \%$ and a sensitivity of $63 \%$ (table 3, cut-off $=$ $1,260 \mathrm{~mm}^{3}$ ). Discrimination of FTLD from AD was achieved with a specificity of $60 \%$ and sensitivity of $63 \%$ (table 3, cut-off $=1,260 \mathrm{~mm}^{3}$ ). Amygdala volumes could discriminate SD from AD with a specificity of $85 \%$ and sensitivity of $86 \%$ (table 3 , cut-off $=1,055 \mathrm{~mm}^{3}$ ); when amygdala asymmetry was included in this regression analysis, sensitivity increased to $100 \%$ whilst keeping the specificity constant.

\section{Discussion}

This study demonstrates that very marked amygdala atrophy occurs in all three syndromic variants of FTLD, as well as in $\mathrm{AD}$. The amygdala volumes and asymmetry scores demonstrated significant differences between FTLD, AD and control groups. In the $\mathrm{AD}$ patients both left and right amygdala volumes were similarly reduced by approximately $20 \%$ of mean control volume. This is consistent with previous studies that have reported between 14 and 44\% loss of amygdala volume in $\mathrm{AD}$ patients $[14,15,20]$. However, the FTLD patients, even when taken together as a group, showed an asymmetric pattern of amygdala atrophy with a striking $41 \%$ loss of volume in the left amygdala, substantially and significantly more than in the AD patients. Atrophy of the right amygdala was also greater in FTLD than AD. Overall, taking the amygdala measurements on their own they had a sensitivity of $63 \%$ and specificity of $60 \%$ in differentiating FTLD and AD.

The most severe amygdala atrophy was seen in the patients with $\mathrm{SD}$, with the mean left amygdala volume being almost $60 \%$ lower than that of the control group, and over $40 \%$ lower in the right amygdala. When amygdala atrophy and asymmetry were taken into account complete separation of the SD patients from the control group was achieved in our sample and more importantly almost complete separation of SD from AD was possible (sensitivity of $100 \%$; specificity of $85 \%$ ). Our findings are in keeping with previous studies demonstrating severe left temporal lobe [7, 10] and amygdala atrophy in SD [8-9]. Amygdala atrophy was also present in the PNFA and
FTD groups, although not greater overall than in the AD group. Unlike the AD patients, the PNFA group appeared to display asymmetry with an estimated $12 \%$ greater leftsided damage. These findings are also in keeping with previous MRI studies that reported asymmetric left frontal and temporal lobe atrophy in PNFA [21, 22] and one report of amygdala atrophy at autopsy in PNFA [23]; however, this is the first MRI study to have looked specifically at the amygdala in PNFA. Amygdala atrophy has previously been reported in frontal variant FTD, but to a lesser degree than in AD [11]. However in that study the AD patients were significantly (11 years) older and had longer mean disease duration than the FTD patients.

Damage to the amygdala has been associated with deficits in both social behaviour and in the recognition of emotion from facial expressions (especially fear) [3, 24]. In this study amygdala atrophy was present in all syndromic variants of FTLD, but the SD group were the most severely affected. Diagnostic criteria imply that FTD patients have earlier and more profound behavioural change than SD patients, however, there is increasing evidence that patients with SD can have significant behavioural dyscontrol, and may be commonly misdiagnosed as FTD $[25,26]$. Behavioural deficits such as changes in eating behaviour, repetitive behaviours, compulsive behaviours and aggression for example are common in SD [5, 27]. Furthermore, deficits in the recognition of emotion from facial expressions have been observed in patients with SD [6], loss of empathy is a major presenting feature of FTLD (especially SD [5]) and the emotional reaction of fear is commonly lost in SD patients [5], suggesting a link to amygdala damage. In fact, an association has been found between the processing of emotions and volume of the amygdala in the temporal variant of FTLD [6]. It is likely that the behavioural deficits commonly found in FTLD, especially in the FTD and SD variants, may involve a complex distributed network of limbic and paralimbic structures, including the amygdala.

This study assessed amygdala atrophy in a large number of FTLD patients $(n=46)$, allowing the assessment of atrophy in all three of the syndromic variants of FTLD. Although the numbers of patients was greater than previously reported volumetric MRI studies in FTLD the syndromic groups were still relatively small. The different clinical presentations seen in FTLD make it difficult to match disease severity accurately to the AD group. MMSE score is particularly reduced in patients with language difficulties. Nevertheless, the AD and FTLD subgroups were well matched for disease duration, the patients in- 
cluded in this study were typical in severity to those presenting to cognitive disorders clinics, and the scans chosen for this study were the first MRIs obtained after the initial presentation at our clinic. Finally, the amygdala is a difficult structure to define and measure accurately due to poor grey-white matter boundary definition and may be associated with relatively large measurement errors. However, in this study the same observer (J.W.) very carefully delineated each amygdala (intra-rater ICC was 0.98 , corresponding to an average $4 \%$ difference in volumes between repeated measures, which compares well with previous studies) and the severity of atrophy was such that very significant differences were found despite potential measurement insensitivity.

This study emphasises that different patterns of amygdala atrophy underlie not just differences between $\mathrm{AD}$ and FTLD but also the different clinical presentations of FTLD. Whilst amygdala measurements provide high sensitivity and specificity for the diagnosis of SD; the variability in amygdala volumes may limit the value of amygdala measurements in the differential diagnosis of FTLD and AD. Patterns of atrophy are usually more informa- tive and discriminatory than measurements of single structures $[8,9,28,29]$. In contrast to the symmetric involvement of the hippocampus and medial temporal lobes seen in $\mathrm{AD}$, disproportionate asymmetric atrophy of the amygdala may indicate FTLD, with left greater than right atrophy suggestive of one of the language variants. Therefore, although amygdala atrophy on its own does not provide complete discriminatory power, disproportionate amygdala atrophy should be considered diagnostically important as part of the general assessment of the pattern of atrophy in patients with cognitive impairment.

\section{Acknowledgements}

The authors would like to thank the subjects and their families for participation, and Dr. John Stevens and Dr. Keith Josephs for their helpful advice and comments. This study was supported by grants from the Medical Research Council, the Alzheimer's Research Trust, the Alzheimer's Society and the special Trustees of UCLH/NHNN.

\section{References}

-1 Harvey RJ, Skelton-Robinson M, Rossor MN: The prevalence and causes of dementia in people under the age of 65 years. J Neurol Neurosurg Psychiatry 2003; 74:1206-1209.

$\checkmark 2$ Neary D, Snowden JS, Gustafson L, Passant U, Stuss D, Black S, Freedman M, Kertesz A, Robert PH, Albert M, Boone K, Miller BL, Cummings J, Benson DF: Frontotemporal lobar degeneration: a consensus on clinical diagnostic criteria. Neurology 1998;51:15461554.

-3 Cummings JL, Duchen LW: Kluver-Bucy syndrome in Pick disease: clinical and pathologic correlations. Neurology 1981;31:1415-1422.

4 Tranel D, Hyman BT: Neuropsychological correlates of bilateral amygdala damage. Arch Neurol 1990;47:349-355.

$\checkmark 5$ Snowden JS, Bathgate D, Varma A, Blackshaw A, Gibbons ZC, Neary D: Distinct behavioural profiles in frontotemporal dementia and semantic dementia. J Neurol Neurosurg Psychiatry 2001;70:323-332.

6 Rosen H, Perry R, Murphy J, Kramer JH, Mychack P, Schuff N, Weiner M, Levenson RW, Miller BL: Emotion comprehension in the temporal variant of frontotemporal dementia. Brain 2002; 125:2286-2295.
7 Mummery CJ, Patterson K, Price CJ, Ashburner J, Frackowiak RSJ, Hodges JR: A voxel-based morphometry study of semantic dementia: relationship between temporal lobe atrophy and semantic memory. Ann Neurol 2000;47:36-45.

$\checkmark 8$ Chan D, Fox NC, Scahill RI, Crum WR, Whitwell J, Leschziner G, Rossor AM, Stevens JM, Cipolotti L, Rossor MN: Patterns of temporal lobe atrophy in semantic dementia and Alzheimer's disease. Ann Neurol 2001;49:433442.

-9 Galton CJ, Patterson K, Graham KS, LambonRalph MA, Williams G, Antoun N, Sahakian BJ, Hodges JR: Differing patterns of temporal atrophy in Alzheimer's disease and semantic dementia. Neurology 2001;57:216-225.

10 Rosen HJ, Gorno-Tempini ML, Goldman WP, Perry R, Schuff N, Weiner M, Feiwell R, Kramer JH, Miller BL: Patterns of brain atrophy in frontotemporal dementia and semantic dementia. Neurology 2002;58:198-208.

11 Boccardi M, Pennanen C, Laakso M, Testa C, Geroldi C, Soininen H, Frisoni GB: Amygdaloid atrophy in frontotemporal dementia and Alzheimer's disease. Neurosci Lett 2002;335: 139-143.

12 Dickson DW: Neuropathology of Pick's disease. Neurology 2001;56(suppl 4):S16-S20.
13 Burns A, Jacoby R, Levy R: Psychiatric phenomena in Alzheimer's disease. IV. Disorders of behaviour. Br J Psychiatry 1990;157:8694.

14 Cuenod CA, Denys A, Michot JL, Jehenson P, Forette F, Kaplan D, Syrota A, Boller F: Amygdala atrophy in Alzheimer's disease: an in vivo magnetic resonance imaging study. Arch Neurol 1993;50:941-945.

15 Laakso MP, Partanen K, Lehtovirta M, Hallikainen M, Hanninen T, Vainio P, Riekkinen P Sr, Soininen H: MRI of amygdala fails to diagnose early Alzheimer's disease. Neuroreport 1995;27:2414-2418.

16 Folstein M, Folstein S, McHughs P: The 'Mini mental state': a practical method for grading the cognitive state of patients for the clinician. J Psychiatr Res 1975;12:189-198.

17 McKhann G, Drachman D, Folstein M, Katzman R, Price D, Stadlan EM: Clinical diagnosis of Alzheimer's disease: report of the NINCDS-ADRDA work group under the auspices of Department of Health and Human Services Task Force on Alzheimer's Disease. Neurology 1984;34:939-944.

18 Freeborough PA, Fox NC, Kitney RI: Interactive algorithms for the segmentation and quantitation of 3-D MRI brain scans. Comput Methods Programs Biomed 1997;53:15-25. 
19 Whitwell JL, Crum WR, Watt HC, Fox NC: Normalization of cerebral volumes by use of intracranial volume: implications for longitudinal quantitative MR imaging. Am J Neuroradiol 2001;22:1483-1489.

-20 Laakso MP, Soininen H, Partanen K, Helkala EL, Hartikainen P, Vainio P, Hallikainen M, Hanninen T, Riekkinen PJ: Volumes of hippocampus, amygdala and frontal lobes in the MRI-based diagnosis of early Alzheimer's disease: correlation with memory functions. J Neural Transm 1995;9:73-86.

-21 Abe K, Ukita H, Yanagihara T: Imaging in primary progressive aphasia. Neuroradiology 1997;39:556-559.

-22 Rosen HJ, Kramer JH, Gorno-Tempini ML, Schuff N, Weiner M, Miller B: Patterns of cerebral atrophy in primary progressive aphasia. Am J Geriatr Psychiatry 2002;10:89-97.
23 Turner RS, Kenyon LC, Trojanowski JQ, Gonatas N, Grossman M: Clinical, neuroimaging, and pathologic features of progressive nonfluent aphasia. Ann Neurology 1996;39:166173.

24 Adolphs R, Tranel D, Damasio H, Damasio A Impaired recognition of emotion in facial expressions following bilateral damage to the human amygdala. Nature 1994;372:669-672.

25 Bozeat S, Gregory CA, Ralph MA, Hodges JR: Which neuropsychiatric and behavioural features distinguish frontal and temporal variants of frontotemporal dementia from Alzheimer's disease? J Neurol Neurosurg Psychiatry 2000; 69:178-186.
26 Thompson SA, Patterson K, Hodges JR: Left/ right asymmetry of atrophy in semantic dementia: behavioral-cognitive implications. Neurology 2003;61:1196-1203.

-27 Lui W, Miller BL, Kramer JH, Rankin K, Wyss-Coray C, Gearhart R, Phengrasamy L, Weiner M, Rosen HJ: Behavioral disorders in the frontal and temporal variants of frontotemporal dementia. Neurology 2004;62:742-748.

28 Boxer A, Rankin K, Miller B, Schuff N, Weiner M, Gorno-Tempini ML, Rosen HJ: Cinguloparietal atrophy distinguishes Alzheimer disease from semantic dementia. Arch Neurol 2003;60:949-956.

29 Boccardi M, Laakso MP, Bresciani L, Galluzzi S, Geroldi C, Beltramello A, Soininen H, Frisoni GB: The MRI pattern of frontal and temporal brain atrophy in fronto-temporal dementia. Neurobiol Aging 2003;24:95-103. 